\title{
Prácticas organizacionales requeridas en el desempeño del profesional de enfermería en la calidad de atención al usuario: emergencia. Hospital General Milagro 2017

Organizational practices required in the performance of the nursing professional in the quality of user care: emergency. Milagro General Hospital 2017.

Milagros Cristina Orozco Reyes. ${ }^{1}$, Jessica Daniela Altamirano Llinin. ${ }^{2} \&$ Holguer Estuardo Romero Urréa. $^{3}$

Recibido: 14-07-2019 / Revisado: 21-08-2019 /Aceptado: 10-09-2019/ Publicado: 04-10-2019

\begin{abstract}
.
DOI: https://doi.org/10.33262/cienciadigital.v3i4.919

The objective of this work is to determine the Organizational Practices Required in the performance of the nursing professional in the quality of attention to the user: Emergency. Hospital General Milagro 2017. The methodology used in this project has a quantitative approach with a retrospective, descriptive and field nature, the tool used was observation. The population of choice were 42 nursing professionals who work in the emergency area, the most important results recorded that after the accreditation there was a positive evolution in relation to patient verification, hand hygiene, reporting of adverse events, training in patient safety, safe surgery, preventive and corrective maintenance of biomedical equipment and venous thromboembolism. The use of dangerous abbreviations, the control of concentrated electrolytes, reprocessing and the prevention of pressure ulcers did not show a significant improvement. The training of infusion pumps showed no problems before or after accreditation. The proposal of the present work consists in the development of protocols and interventions for the health promotion, training and the determination of management indicators that allow to take better control of the nursing professional's protocols. It is concluded that the
\end{abstract}

\footnotetext{
${ }^{1}$ Universidad Estatal de Milagro, Milagro, Ecuador, m.orozco.mo@gmail.com

${ }^{2}$ Universidad Estatal de Milagro, Milagro, Ecuador, jaltamirano1987@ hotmail.com

${ }^{3}$ Universidad Estatal de Milagro, Milagro, Ecuador, hromerou@unemi.edu.ec
} 
international accreditation has had a positive effect within the Required Organizational Practices and performance of the nursing staff in triage, procedure, resuscitation and observation of the emergency area

Keywords: International Certifications, Nursing Care, Organizational Practices Required

\section{Resumen.}

El objetivo del presente trabajo es determinar las Prácticas Organizacionales Requeridas en el desempeño del profesional de enfermería en la calidad de atención al usuario: Emergencia. Hospital General Milagro 2017. La metodología que se emplea en este proyecto tiene un enfoque cuantitativo con un carácter retrospectivo, descriptivo y de campo, la herramienta utilizada fue la observación. La población de elección fueron 42 profesionales de enfermería que laboran en el área de emergencia, los resultados obtenidos más importantes registran que luego de la acreditación se presentó una evolución positiva con relación a la verificación del paciente, higiene de manos, reporte de eventos adversos, capacitación de seguridad al paciente, cirugía segura, mantenimiento preventivo y correctivo de equipos biomédicos y tromboembolismo venoso. El uso de abreviaturas peligrosas, el control de electrolitos concentrados, la reprocesamiento y la prevención de úlceras por presión no observó una mejora significativa. El entrenamiento de bombas de infusión no mostró problemas antes ni luego de la acreditación. La propuesta del presente trabajo consiste en el desarrollo de protocolos e intervenciones para la promoción de salud, capacitaciones y el determinar indicadores de gestión que permitan llevar un mejor control de los protocolos del profesional de enfermería. Se concluye que la acreditación internacional ha tenido un efecto positivo dentro de las Prácticas Organizacionales Requeridas y desempeño del personal de enfermería en triage, procedimiento, reanimación y observación del área de emergencia.

Palabras claves: Certificaciones Internacionales, Cuidados de Enfermería, Prácticas Organizacionales Requeridas.

\section{Introducción.}

El inicio de las practicas organizacionales requeridas en el desempeño del profesional de enfermería durante el proceso de Certificación Internacional a nivel Hospitalario en la República de Ecuador son considerados por el Ministerios de Salud Pública (MSP) como un mecanismo más para mejorar la calidad de los servicios de salud, garantizando la accesibilidad y equidad para el mejoramiento del nivel de salud de la población. 
Posteriormente y en la actualidad el Consejo Nacional de Acreditación del Ecuador (CONAH) con el apoyo de otras entidades del sector salud, se dedican a la evaluación, implementación y calificación de la calidad de los servicios de niveles hospitalarios que funcionan en el país. De esta manera se observa que los avances en el campo de la Acreditación de las instituciones de salud han estado enmarcados en el desarrollo de las políticas dirigidas directamente desde el Ministerio de Salud Pública y con los que se busca integrar a todos los entes del sector salud de este país.

La saturación de los servicios de emergencia de los hospitales públicos es también una realidad, lo que refleja las deficiencias en la coordinación entre estos y los servicios de atención primaria en primer lugar, secundada por el hecho de que la población que demanda estos servicios la ven como una forma de atención más especializada y más rápido para observar su problema de salud como una situación urgente. El triage constituye una herramienta que permite de forma satisfactoria clasificar al paciente de acuerdo a la emergencia que tenga que recibir atención de salud, la misma que inicialmente fue creada para ser aplicada en situaciones de catástrofe y en la actualidad es aplicada para emergencias hospitalarias.

La problemática observada en el área de emergencia sobre los procesos que conducen al fomento de una mejor atención del usuario es que no tenían lineamientos, ni parámetros establecidos, peor aún normativas específicas, y se llevaban a cabo de la manera empírica, por todos los profesionales que integran el equipo interdisciplinario, causando inconvenientes en los tiempos de espera de los usuarios para la atención médica, el control de los medicamentos de alto riesgo, comunicación deficiente de sucesos de eventos adversos, capacitación de seguridad al paciente, el desconocimiento sobre las abreviaturas peligrosas y el reprocesamiento de insumos, forma incorrecta de higiene de manos, verificación de los datos del cliente, control de electrolitos concentrados, control deficiente tromboembolismo venoso, prevención de úlceras por presión, cirugía segura, entrenamiento de bombas de infusión y riesgo de caída, no contar con un mantenimiento preventivo y correctivo de equipos biomédicos.

En el triage y el área de procedimientos el profesional de enfermería por la gran demanda de usuarios que exigen la atención médica, tampoco se cuenta un manual de procedimientos o técnicas específicas para evidenciar un adecuado proceso; no se realiza una adecuada higiene de manos, la verificación de datos está incompleta. En el área de Reanimación y observación se repiten todos los conflictos detallados en las dependencias anteriormente mencionadas. Estas falencias determinan la necesidad de diseñar diversos protocolos de Enfermería que servirán para mejorar la calidad de atención en el servicio.

El propósito de esta investigación es determinar las Prácticas Organizacionales Requeridas en el desempeño del profesional de enfermería en la calidad de atención al usuario en el área de Emergencia del Hospital General Milagro. Determinando las Prácticas Organizacionales 
Requeridas que cumple el profesional de enfermería en la dependencia de Triage y procedimientos del área de emergencia; así mismo se conseguirá determinar las Prácticas Organizacionales Requeridas que cumple el profesional de enfermería en la dependencia de reanimación y observación el área de emergencia. A su vez se establecerá un diseño de Protocolos de Enfermería para solucionar el problema que generan los resultados de la investigación en el profesional de enfermería del área de emergencia.

\section{Metodologia.}

Es cuantitativo, de campo, prospectivo, de corte longitudinal de tipo: diseño de análisis evolutivo, ya que se tomó una línea base y se analizaron los cambios a través del tiempo durante 4 meses, obteniendo datos cada mes, para evaluar a todos los profesionales. De los cuales se realizó una función aritmética que permitió obtener un resultado que se comparó con la línea de base y se obtuvo el resultado descrito y que nos servirá para dar una explicación y solución a la problemática de esta investigación.

Se midió el fenómeno utilizando la estadística, fue secuencial y analizó una realidad objetiva para así generar los resultados obtenidos mediante la ficha de observación al profesional de enfermería. Esto se realizó para conocer si las Prácticas Organizacionales Requeridas influyen en el desempeño del profesional de enfermería de la unidad de salud anteriormente mencionada.

Se estableció una línea base cuando se certificó todo el hospital General Milagro, donde se evaluaron las variables de investigación en el área de emergencia; cabe destacar que, durante el proceso de certificación el área y la institución se fueron capacitando, evaluando mensualmente el avance en el desempeño del personal.

La investigación se efectuó en el Hospital General Milagro, la misma que contó con los 42 profesionales en Enfermería, realizando una ficha de observación a los mismos del área de Emergencia.

Las variables que intervinieron en el estudio fueron Prácticas Organizacionales Requeridas y profesional de enfermería. Las dimensiones de las POR fueron verificación del paciente, eventos adversos, higiene de manos, abreviaturas peligrosas, capacitación de seguridad al paciente, control de electrolitos concentrados, cirugía segura, reprocesamiento, prevención de úlceras por presión, mantenimiento preventivo y correctivo de equipos biomédicos, tromboembolismo venoso.

La forma de evaluación fue llenando la ficha que contiene las opciones de cumplimiento y no cumplimiento, con lo cual se establecieron porcentajes de cumplimiento de parte de los profesionales de enfermería. Se mostraron las diferencias porcentuales de las POR para evaluar si ha existido una diferencia significativa entre las prácticas. 
Los datos obtenidos en los instrumentos aplicados fueron tabulados y resumidos en tablas estadísticas, desarrollados en Excel.

\section{Resultados.}

Las Prácticas Organizacionales Requeridas en el desempeño del profesional de enfermería en la calidad de atención al usuario: Emergencia. Hospital General Milagro 2017, fueron las siguientes: verificación del cliente, higiene de manos, mantenimiento preventivo y correctivo de equipos, abreviaturas peligrosas, capacitación de seguridad al paciente, eventos adversos, control de electrolitos concentrados, cirugía segura, reprocesamiento, prevención de úlceras por presión, riesgo de caídas, tromboembolismo venoso, entrenamiento de bombas de infusión.

Es importante destacar que todos los grupos que formaron parte de la encuesta como requisito indispensable han estado en contacto con los procedimientos, protocolos e implementación durante la acreditación internacional y están relacionados con la evolución y evaluación de los mismos en el Hospital General de Milagro.

Tabla 1. Por triage y procedimientos área de emergencia

\begin{tabular}{llll}
\hline POR & Opción & Frecuencia & Porcentaje \\
\hline Verificación del paciente. & - Cumplimiento & -18 & $-81.8 \%$ \\
& - No cumplimiento & -4 & $-18.2 \%$ \\
Higiene de manos & - Cumplimiento & -22 & $-100 \%$ \\
& - No cumplimiento & & \\
$\begin{array}{l}\text { Mantenimiento } \\
\text { preventivo y correctivo } \\
\text { de equipos biomédicos. }\end{array}$ & - Cumplimiento & -18 & $-81.8 \%$ \\
\hline
\end{tabular}

Nota: Se muestra en porcentaje los resultados obtenidos en cuanto a la ejecución de procesos institucionales para la mejora del área de Enfermería

Fuente: Hospital General Milagro

Elaboración: Grupo de investigación.

En las POR del triage y procedimientos del área de emergencia se evidenció que la verificación del paciente tuvo un cumplimiento del $81.8 \%$, eventos adversos $77.3 \%$, higiene de manos $100 \%$, capacitación de seguridad al paciente $90.9 \%$, reprocesamiento $72.7 \%$, mantenimiento preventivo y correctivo de equipos biomédicos $81.8 \%$, mientras que el entrenamiento de bombas de infusión $100.0 \%$. Las abreviaturas peligrosas, cirugía segura, prevención de úlceras por presión y tromboembolismo venoso no aplicaron para el triage y procedimiento del área de emergencia. 
El propósito de Enfermería es brindar el mejor nivel de cuidado, no menos que excelente en cuanto a la calidad técnica, humana y ética; lo que implica un profundo compromiso profesional con la actualización de conocimientos científicos con disciplina y responsabilidad de acuerdo a lo establecido por Jean Watson en la teoría del cuidado (Endocolombia, 2012); cabe destacar que una vez realizada la certificación internacional los valores obtenidos fueron satisfactorios como se puede observar en la tabla de procesos institucionales para la mejora del área de enfermería.

En el Hospital General Milagro se observa que se aplica el manual de procedimientos con los debidos lineamientos utilizando el manual de diagnóstico, la enfermera gestora favorece una cultura centrada en el cuidado de la persona, ya que es una forma dinámica y sistematizada capaz de brindar los respectivos cuidados enfermeros y lograr identificar si existe una gestión por procesos de acuerdo a lo establecido en la teoría del cuidado (Endocolombia, 2012).

A continuación, se muestra el cumplimiento de las POR para la dependencia de reanimación y observación del área de emergencia:

Tabla 2. Por reanimación y observación área de emergencia

\begin{tabular}{llll}
\hline POR & Opción & Frecuencia & Porcentaje \\
\hline Verificación del paciente. & - Cumplimiento & -16 & $-80.0 \%$ \\
& - No cumplimiento & -4 & $-20.0 \%$ \\
Eventos adversos & - Cumplimiento & -15 & $-75.0 \%$ \\
& - No cumplimiento & -5 & $-25.0 \%$ \\
Higiene de manos & - Cumplimiento & -20 & $-100.0 \%$ \\
& - No cumplimiento & & \\
Abreviaturas peligrosas. & - Cumplimiento & -11 & $-55.0 \%$ \\
& - No cumplimiento & -9 & $-45.0 \%$ \\
$\begin{array}{l}\text { Capacitación de seguridad } \\
\text { al paciente. }\end{array}$ & - Cumplimiento & -19 & $-95.0 \%$ \\
$\begin{array}{l}\text { Control de electrolitos } \\
\text { concentrados. }\end{array}$ & - No cumplimiento & -1 & $-5.0 \%$ \\
Cirugía segura. & - Cumplimiento & -15 & $-75.0 \%$ \\
& - No cumplimiento & -5 & $-25.0 \%$ \\
Reprocesamiento. & - Cumplimiento & -16 & $-80.0 \%$ \\
\hline & - No cumplimiento & -4 & $-20.0 \%$ \\
\hline
\end{tabular}




\begin{tabular}{llll}
\hline & - No cumplimiento & -4 & $-20.0 \%$ \\
$\begin{array}{l}\text { Prevención de úlceras por } \\
\text { presión. }\end{array}$ & - Cumplimiento & -10 & $-50.0 \%$ \\
& - No cumplimiento & -10 & $-50.0 \%$ \\
$\begin{array}{l}\text { Mantenimiento preventivo } \\
\text { y correctivo de equipos } \\
\text { biomédicos. }\end{array}$ & - Cumplimiento & -18 & $-90.0 \%$ \\
$\begin{array}{l}\text { Tromboembolismo venoso } \\
\text { - No cumplimiento }\end{array}$ & - Cumplimiento & -16 & $-10.0 \%$ \\
& - No cumplimiento & -4 & $-80.0 \%$ \\
$\begin{array}{l}\text { Entrenamiento de bombas } \\
\text { de infusión }\end{array}$ & - Cumplimiento & -20 & $-20.0 \%$ \\
& - No cumplimiento & & $-100.0 \%$ \\
\hline
\end{tabular}

Nota: Se muestra en porcentaje los resultados obtenidos en cuanto a la ejecución de procesos institucionales para la mejora del área de Enfermería

Fuente: Hospital General Milagro

Elaboración: Grupo de investigación

En los resultados se evidencia que la verificación del paciente en la dependencia de reanimación y observación mostró un cumplimiento del $80.0 \%$, en eventos adversos $75.0 \%$, higiene de manos $100.0 \%$, abreviaturas peligrosas $55.0 \%$, capacitación de seguridad al paciente $95.0 \%$, control de electrolitos concentrados $75.0 \%$, cirugía segura $80.0 \%$, reprocesamiento $80.0 \%$, prevención de úlceras por presión $50.0 \%$, mantenimiento preventivo $\mathrm{y}$ correctivo de equipos biomédicos $90.0 \%$, tromboembolismo venoso con el $80.0 \%$ y entrenamiento de bombas de infusión con el $100.0 \%$ de cumplimiento.

\section{Discusión}

Las Prácticas Organizacionales Requeridas fueron evaluadas antes y después de la certificación. Los resultados permitieron evidenciar que antes de la certificación existía un 83.3\% de incumplimiento en la verificación del paciente, el 81.0\% no cumplía con la higiene de manos, mientras que el $52.4 \%$ no cumplía con el reporte de eventos adversos.

Luego de la acreditación se observó una evolución con respecto a estos aspectos. La verificación del paciente tuvo un cumplimiento del $81.0 \%$, logrando una mejora del $64.3 \%$ con respecto a antes de la acreditación. La higiene de manos evidenció un cumplimiento del $76.2 \%$, lo que significó una mejora del 57.2\%; en tanto que el reporte de eventos adversos mostró un $100 \%$ de cumplimiento, lo cual evidenció una mejora del $52.4 \%$ del personal. En términos generales se considera que estas Prácticas Organizacionales Requeridas han tenido una mejora significativa luego de la acreditación del hospital; sin embargo, se debe trabajar para lograr el cumplimiento del $100 \%$ de las prácticas. 
En lo que respecta al uso de abreviaturas peligrosas hubo un $45 \%$ de personas que mostraron problemas en este aspecto antes de la acreditación, luego de ella se obtuvo una mejora del $10 \%$ lo cual no fue suficiente para mostrar una evolución significativa. En la capacitación de seguridad al paciente se observó que hubo un cumplimiento del 92.9\% del personal, la mejora observada fue del 14.3\%, siendo positivo para la institución.

El control de electrolitos concentrados mostró un cumplimiento del $75.0 \%$, teniendo una mejora del $25 \%$ con respecto a antes de la acreditación que no logra ser significativa para lo que busca el hospital; no obstante, a pesar de ser positivo, muestra que existe una cantidad importante de personal que no está cumpliendo con este aspecto. La cirugía segura tuvo un cumplimiento del $80.0 \%$, que comparado al $50 \%$ presentado antes de la acreditación presenta una evolución, aunque no del $100 \%$ que es lo ideal a alcanzar. La reprocesamiento pasó de un $73.8 \%$ a un $76.2 \%$ evidenciando una mejora del $2.4 \%$ que no es estadísticamente positiva para el hospital, lo que demuestra que este proceso debe ser mejorado para lograr alcanzar el $100 \%$.

La prevención de úlceras por presión después de la acreditación mostró un incumplimiento del 50\%, que no reflejó ninguna evolución con respecto a antes de la acreditación. El mantenimiento preventivo y correctivo de equipos biomédicos pasó del $19.0 \%$ antes de la acreditación a un $85.7 \%$ después de la acreditación lo que significó una mejora del $66.7 \%$. El tromboembolismo venoso pasó de un $15.0 \%$ de cumplimiento a un $80.0 \%$, evidenciando una evolución del $65.05 \%$, mientras que el entrenamiento de bombas de infusión evidenció un $100 \%$ de cumplimiento. En términos generales, se considera que la acreditación internacional ha tenido un efecto positivo dentro de las POR y desempeño del personal de enfermería en triage, procedimiento, reanimación y observación del área de emergencia.

Algunos factores que llevan a las instituciones a buscar la acreditación son la existencia de estímulos específicos, que pueden ser proporcionados como: instalaciones de contratación, mejoras en las condiciones impuestas por los aseguradores, reducción de los aranceles de importación, entre otros. Sin embargo, la mayoría de los proveedores comparten la idea de que estos estímulos deben ser de naturaleza económica.

Por otro lado, la acreditación es un potente motor de mejora y calidad continua, fomentando una adecuada cultura organizacional que involucre a todo el personal de la organización en el desarrollo de esta metodología, fomenta la satisfacción de los usuarios y mejores resultados de salud convergente en el prestigio institucional como se pudo evidenciar en las fichas de observación aplicadas al personal de enfermería.

\section{Conclusiones.}

- Las Prácticas Organizacionales Requeridas en el desempeño del profesional de enfermería en la calidad de atención al usuario en el área de emergencia del Hospital 
General Milagro son 3 en la dependencia de traige y procedimientos: Verificación del paciente, higiene de manos y mantenimiento preventivo y correctivo de equipos biomédicos, en la dependencia de Reanimación y Observación incluyendo los 3 ítems anteriores Abreviaturas peligrosas, capacitación de seguridad al paciente, eventos adversos, control de electrolitos concentrados, cirugía segura, reprocesamiento, prevención de ulceras por presión, riesgo de caídas, tromboembolismo venoso, entrenamiento de bombas de infusión.

- En la dependencia de reanimación y observación del área de emergencia, se concluye que la verificación del paciente, eventos adversos, higiene de manos, abreviaturas peligrosas, capacitación de seguridad al paciente, control de electrolitos concentrados, cirugía segura, reprocesamiento, prevención de úlceras por presión, mantenimiento preventivo y correctivo de equipos biomédicos, tromboembolismo venoso y entrenamiento de bombas de infusión sí se cumplieron en las dependencias nombradas.

- La propuesta para solucionar el problema que generan los resultados de la investigación en el profesional de enfermería del área de emergencia, se debe basar en protocolos e intervenciones del personal para el mejoramiento en la promoción de la salud en el Hospital General Milagro, se debe además fortalecer la capacitación del profesional de enfermería para dar charlas de promoción de la salud a la población de Milagro y sus alrededores, además que se deben determinar indicadores de gestión mediante métodos teóricos, que permitan llevar un mejor control de los protocolos del profesional de enfermería en el área de Emergencia del Hospital General Milagro.

\section{Referencias bibliográficas.}

Álvarez, F. (2017). Calidad y auditoría en salud. Bogotá: Ecoe ediciones.

Asociación canadiense de Salud Pública. (2016). Enfocando la salud. La salud pública en la reestructuración de los servicios de salud. Canadá: Documento de estudio, Consejo Directivo.

Beltrán Barahona, M., \& Remache López, C. (2016). Impacto del proceso de acreditación canadiense en el cuidado de enfermería en el área de cirugía del Hospital José María Velasco Ibarra de la ciudad del tena periodo octubre 2015 - febrero 2016 . Obtenido de http://www.dspace.uce.edu.ec/bitstream/25000/6231/1/T-UCE-0006-031.pdf

Endocolombia. (2012). Teoría del Cuidado Humano de Jean Watson. Recuperado el 22 de Septiembre de 2015, de http://encolombia.com/medicina/revistasmedicas/enfermeria/ve-104/trabajoslibres1/ 
Figueiredo Manzo, B., Couto Ribeiro, H., Menezes Brito, M., \& Alves, M. (2012). La enfermería en hospital en proceso de acreditación: su papel y las. Obtenido de http://www.scielo.br/pdf/rlae/v20n1/es_20.pdf.

Jiménez, L. y Montero, J. (2017). Compendio de medicina de urgencias. Barcelona: Elsevier.

Keith, S. y Humphries, R. (2009). Diagnóstico y Tratamiento de urgencias. México: Editorial El Manual moderno.

Torrent, R. L., Sánchez Palacios, M. S., Santana Cabrera, L., Cobian Martínez, J. L., \& García del Rosario, C. (2010). Gestión de la calidad de una unidad de cuidados intensivo. Implementación de la Norma ISO 9001.2008. Medicina Intensiva. Punto de vista.

Torres Velez, D., \& Viejo Toro, S. (2015). Cumplimiento de la seguridad del paciente basado en la acreditación canadiense en el área de observación emergencia del hospital universitario de Guayaquil durante el periodo de mayo a septiembre del 2015. Obtenido de http://repositorio.ucsg.edu.ec/bitstream/3317/4147/1/T-UCSG-PREMED-ENF-209.pdf.

Amaro Cano, María del Carmen. (2004). Florence Nightingale, la primera gran teórica de enfermería. Revista Cubana de Enfermería, 20(3) Recuperado en 19 de julio de 2019, de http://scielo.sld.cu/scielo.php?script=sci_arttext\&pid=S086403192004000300009\&lng=es\&tlng=es.

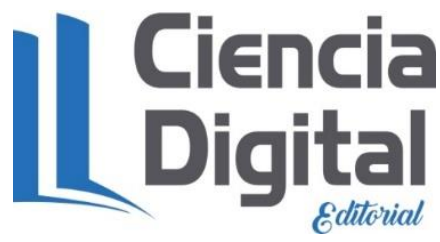


PARA CITAR EL ARTÍCULO INDEXADO.

Orozco Reyes, M., Altamirano Llinin, J., \& Romero Urréa, H. (2019). Prácticas organizacionales requeridas en el desempeño del profesional de enfermería en la calidad de atención al usuario: emergencia. Hospital General Milagro 2017. Ciencia Digital, 3(4), 44-54. https://doi.org/10.33262/cienciadigital.v3i4.919

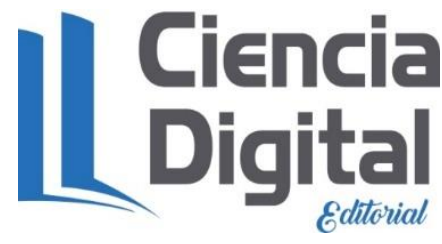

El artículo que se publica es de exclusiva responsabilidad de los autores y no necesariamente reflejan el pensamiento de la Revista Ciencia Digital.

El artículo queda en propiedad de la revista y, por tanto, su publicación parcial y/o total en otro medio tiene que ser autorizado por el director de la Revista Ciencia Digital.
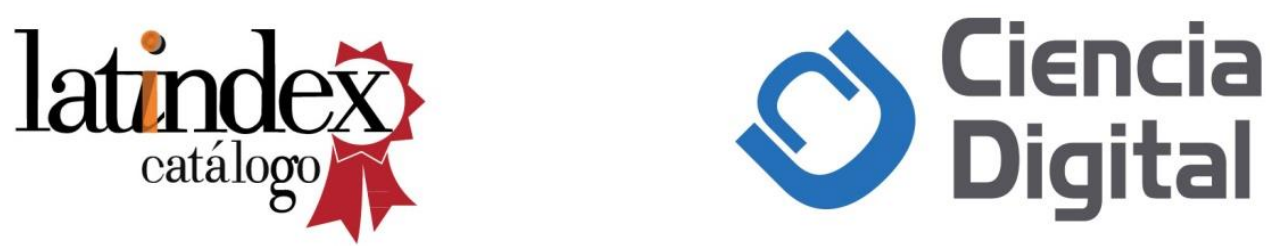